\title{
High-Performance Concrete Compressive Strength Prediction Based Weighted Support Vector Machines
}

\author{
Rguig Mustapha*, EL Aroussi Mohamed** \\ *(LETI Laboratory, EHTP Casablanca) \\ **(LETI Laboratory, EHTP Casablanca)
}

\begin{abstract}
Concrete is the safest and sustainable construction material which is most widely used in the world as it provides superior fire resistance, gains strength over time and gives an extremely long service life. Unfortunately high performance concrete is undoubtedly one of the most innovative materials in construction. Its Designing involves the process of selecting suitable ingredients of concrete (water, cement, fine and aggregates and a number of additives like mineral and chemical admixture) and determining their relative amounts with the objective of producing a high performance concrete of the required, strength, durability, and workability as economically as possible. Their proportions have a high influence on the final strength of the product. These relations do not seem to follow a mathematical formula and yet their knowledge is crucial to optimize the quantities of raw materials used in the manufacture of high performance concrete. Therefore, it would be important to have a tool to numerically model such relationships, even before processing. In this aspect the main purpose of this paper is to predict the compressive strength of the high performance concrete by using classification algorithms. For building these models, training and testing using the available experimental results for 1030 specimens produced with 8 different mixture proportions are used. The result from this study suggests that weighted Support Vector Machines (wSVM) based models perform remarkably well in predicting the compressive strength of the concrete mix.
\end{abstract}

Keywords: HPC (High-Performance Concrete), Weighted Support Vector Machines, Compressive Strength Prediction

\section{INTRODUCTION}

In [1] yeh et al stat that High-performance concrete (HPC) is a new type of concrete used in the construction industry. HPC works better in terms of performance characteristics and uniformity characteristics than high-strength concrete [2,3]. Prediction of High-performance concrete strength is important for concrete construction as it gives an idea about the time for concrete form removal, project scheduling and quality control. The major difference between HPC and conventional concrete is essentially the use of mineral and chemical admixtures [4,5]. Therefore, Apart from the four conventional cement ingredients, Portland Cement (PC), water, fine aggregates, and coarse aggregates, HPC further incorporates cementitious materials, fly ash, blast furnace slag, and a chemical admixture [1]. These additional ingredients make HPC mix proportion calculations and HPC behavior modeling significantly more complicated than corresponding processes for conventional cement. Chou et al [6] stated that certain properties of HPC are not fully understood since the relationship between ingredients and concrete properties is highly nonlinear. Therefore, traditional model of concrete properties is inadequate for analyzing HPC compressive strength.
There are popular methods of mix proportion of HPC such method proposed by $[7,8,9]$ among other methods [10]. However, to obtain required mix proportions of HPC most commonly based on trial mixes as stated in relevant standards, experience, and rules of thumb approach $[11,12]$.

Twenty-eighth day compressive strength is the most widely used objective function in the mixture design. However, as pointed out previously, the result depends on ingredient combinations and proportions, mixing techniques and other factors that must be controlled during manufacturing. Kasperkicz et al. [13] stated that the introduction of new ingredients and technologies implies that the number of parameters for HPC mix design may extend to 10-, 20- or even higher dimensional decision space numbers. Waiting 28 days to get 28 day compression strength is time consuming and not a common practice in the construction industry. Therefore, many researchers have worked to establish prediction tools able to obtain an early determination of compressive strength, ideally well before concrete is laid down at a construction site. Prediction of concrete compressive strength is one area of active research in the civil engineering field, and a considerable number of relevant studies have been carried out over the past 30 years. Zain and Abd [14] attempted to categorize methods into three 
types, i.e., those using statistical techniques, computational modeling and artificial neural networks. Statistical techniques represent a conventional approach, and are used primarily to predict conventional concrete compressive strength by establishing linear and nonlinear regression equations. Several approaches using regression functions have been proposed for predicting the concrete strength $[15,16,17,18]$.

Traditional modeling approaches are based on empirical relationships derived from the experimental data. The approach starts with an analytical equation assumption, followed by regression analysis that employs limited experimental data to determine an unknown coefficient. While many regression models have been suggested, obtaining a suitable regression equation is not an easy task. Moreover in this prediction effort, the early compressive strength at 6hour, 1-day and 3-day is usually embodied in a prediction equation that necessitates some time delay in prediction [19]. Furthermore, for HPC, where the number of influencing factors is greater than for conventional concrete, this regression model is neither suitable nor adequate to predict compressive strength [20]. As traditional methods handle complex non-linear and uncertain materials (like HPC) poorly, many researchers have sought better prediction tools. Many studies have proposed artificial neural networks (ANNs) and ANN variations to map non-linear relationships among factors of influence on 28-day HPC compressive strength. Kasperkic z et al. [13] proposed an artificial neural network of the fuzzy-ATMAP to predict HPC strength properties. It was found that concrete property prediction could be effectively modeled using a neural system without being affected by data complexity, incompleteness, or incoherence. In 1998, Yeh et al [1] demonstrated the superiority of ANNs in predicting HPC compressive strength that produced better results than regression analysis. Yeh also showed how easily ANNs could adapt to different numerical experiment settings in order to review the effect on the concrete mix of each variable proportion. Akkurt et al. [21] also noted the use of fuzzy logic to predict concrete compressive strength.

Within last decade, machine learning and AI are attracting increasing attention in academic and empirical fields for their potential application to civil engineering problems [22]. In the field of civil engineering, much research has focused on prediction techniques. Therefore researchers have explored the potential of artificial neural networks (ANNs), a nonlinear modeling approach, in predicting the compressive strength of the concrete due to its ability to learn input-output relation for any complex proble $m$ in an efficient way.
Several work reported the use of neural network based modeling approach in predicting the concrete strength (Sergio Lai and Mauro Serra, 1997; Yeh, 1998a, 1998b, 1999; Kasperkiewicz et al., 1995; Sebastia et al., 2003;

Kim et al., 2004; Dias and Pooliyadda, 2001; Nehdi et al.,2001; Oh et al., 1999). In most of the studies a back propagation neural network was used. A neural network model requires no functional relationship among the variables, as is the case with most of other regression analysis techniques. A neural network based modeling algorith $m$ requires setting up of different learning parameters (like learning rate, momentum), the optimal number of nodes in the hidden layer and the number of hidden layers so as to have a less complex network with a relatively better generalization capability. A large number of training iterations may force ANN to over train, which may affect the predicting capabilities of the model.

Also many papers have reported on hybrid techniques that are able to predict HPC to a high degree of accuracy (Cheng et al., 2012; Peng et al., 2009; Yeh, 1999).

Within last few years, another modeling technique called Support Vector Machines (SVMs) (Vapnik 1995) is being applied in the field of civil engineering (Dibike et. al. 2001, Pal and Mather 2003). SVM, which represents a new AI technique, has been shown to deliver comparable or higher performance than traditional learning machines and has been introduced as a powerful tool to solve classification and regression problems [23,24]. In [25] Gupta investigates the potential of support vector machines in predicting the compressive strength of high strength concrete. Radial bas is function (RBF) and polynomial kernels are used with support vector machines. However, SVM presents several inherent shortcomings. Firstly, SVM is unable to provide high prediction accuracy for either the penalty parameter (C) or kernel parameter settings. Secondly, SVM considers all training data points equally in order to establish the decision surface. Therefore, Ling and Wang [26] proposed a modified version of SVM, known as fuzzy SVM (FSVM) or weighted SVM (wSVM), to weight all training data points in order to allow different input points to contribute differently to the learning decision surface. The objective of present study is to examine the potential of support vector machines (wSVMs) for predicting the compressive strength of high strength concrete.

We evaluate the effectiveness of the proposed method using an experimental database originally generated by Yeh [27] and posted to the University of California, Irvine machine learning repository website. To verify and validate the proposed system, wSVM performance was 
compared against original SVMs and has shown better recognition performance.

The rest of this paper is organized as follows. A Brief introduction to SVM and weighted SVMs is introduced in section 2. Experimental results are presented and discussed in Section 3. Finally, conclusions are stated in the last section.

\section{BRIEF INTRODUCTION TO SVM AND WEIGHTED SVMS}

\section{SVM}

This section addresses support vector machines (SVM), the classifier used in this dissertation for the detection of concrete surface cracks.

An SVM classifier, just like other learning algorithms, is composed by training and testing stages [28]. In the training stage the selected features are extracted and typically mapped into a higher dimensional space in order to efficiently separate crack features from non-crack features. Since the ground-truth of the training set is supplied, the features that correspond to cracks and to non-cracks can be determined. Then, as illustrated in Fig.1, SVM selects the set of points in each class (support vectors) that are the nearest to the other class and through them computes a hyperplane that separates the two classes, being as far as possible from the support vectors. This hyperplane is often call maximum-marg in hyperplane and makes SVM robust.

Once the system has been trained, the following phase is the testing stage. In this stage each testing sample is classified as belonging to one of the two pattern classes. For that the testing set features are mapped into the same dimensional space produced in the training stage and, according to the hyperplane side they fall, the corresponding pattern class is attributed. Finally, the classifier accuracy can be evaluated by comparison against a set of manually labeled data.

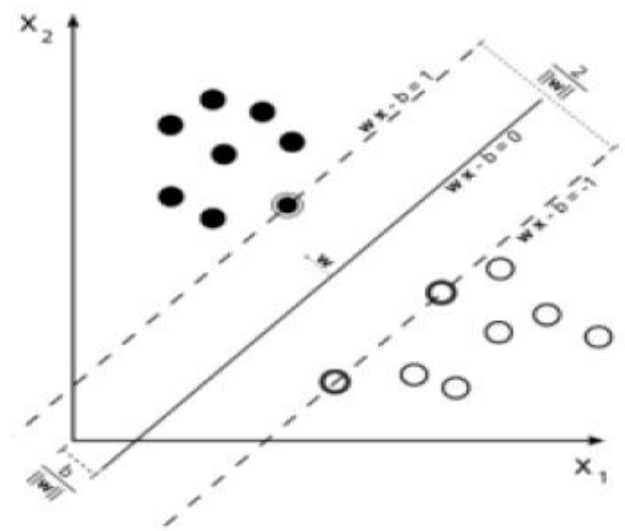

Fig. 1. SVM feature space example that selects the support vectors to separate the two pattern classes through a hyperplane.
The example illustrated in Fig.8is very simple and there is no need to map the extracted features into a higher dimensional space since they can be easily separated by a hyperplane. However the typical case is much more complex and the two classes are often mixed, being necessary to map the features to separate better the two classes. Note that in Fig.1 several different hyperplanes could separate the two classes. However the hyperplane computed, tries to be as far as possible from the support vectors.

Given a training set of instance-label pairs $\left\{x_{i}, y_{i}\right\}, i=1, \ldots, 1$ where $x_{i} \in R$ and $y \in\{-1,1\}^{1}$, the support vector machines require the solution of the following optimization problem:

$$
\min _{(\omega, b, \varepsilon)} \frac{1}{2} \omega^{T} \omega+C \sum_{i=1}^{l} \varepsilon_{i}
$$

Subject to:

$$
\left(\omega^{T} \phi\left(x_{i}\right)+b\right) \geq 1-\varepsilon_{i}, \varepsilon_{i} \geq 0
$$

Here training vectors xi are mapped into a higher (maybe infinite) dimensional space by the function $\Phi$. Then SVM finds a linear separating hyper plane with the maximal marg in in this higher dimensional space. $\mathrm{C}>0$ is the penalty parameter of the error term. We can define, $\mathrm{K}(\mathrm{xi}, \mathrm{xj})=$ $\Phi(\mathrm{xi}) \mathrm{T} \Phi(\mathrm{xj})$ called the kernel function. Though new kernels are being proposed by researchers, the most common four basic kernels are: Linear, Polynomial, Radial basis function (RBF) and Sig moid.

- Linear: $\mathrm{K}(\mathrm{xi}, \mathrm{xj})=\mathrm{xi}^{\mathrm{T}} \mathrm{xj}$

- Polynomial :

$\mathrm{K}\left(\mathrm{x}_{\mathrm{i}}, \mathrm{x}_{\mathrm{j}}\right)=\left(\Upsilon \mathrm{x}_{\mathrm{i}} \mathrm{x}_{\mathrm{j}}+\mathrm{r}\right)^{\mathrm{d}}, \Upsilon>0$

- Radial basis function $(\mathrm{RBF})$ :

$\mathrm{K}\left(\mathrm{x}_{\mathrm{i}}, \mathrm{x}_{\mathrm{j}}\right)=\exp \left(-\Upsilon\left\|\mathrm{x}_{\mathrm{i}}-\mathrm{x}_{\mathrm{j}}\right\|^{2}\right), \Upsilon>0$

- $\quad$ Sig moid : $K\left(x_{i}, x_{j}\right)=\tanh \left(\Upsilon x^{T}{ }^{T} x j+r\right)$

Where $\Upsilon, \mathrm{r}$ and $\mathrm{d}$ correspond to kernel parameters that can be defined or estimated.

\section{WeightedSVMS}

The weighted support vector machine (WSVM) is a SVM adaptation to the cost sensitive learning framework. This supervised learning technique has been successfully applicable for imbalanced classification.

The term "weighted support vector machines" (wSVMs) was proposed by Fan and Ramamo hanarao [29] as a synonym for Fuzzy Support Vector Machines (FSVMs) to draw attention to the effective weighting of fuzzy me mberships at each FSVM training point.

Fan and Ramamo hanarao [29] stated that different input vectors make different contributions to the learning of decision surface. Thus, the important issue in training weighted SVMs is how to develop a reliable weighting model to reflect the true 
noise distribution in the training data. Fan and Ramamo hanarao [29] developed emerg ing patterns (EPs) to weight the training data. Lin and Wang [30] developed FSVMs to enhance support vector machine (SVM) abilities to reduce the effects of outliers and noise in data points. While SVMs a recent AI paradig m developed by Vapnik [30] that has been used in a wide range of applications, treat all training points of a given class uniformly, training points in many real world applications bear different importance weightings for classification on purposes. To solve this problem, Lin and Wang [31] applied a fuzzy member to each SVM input point, thus allowing different input points to contribute differently to the learning decision surface. In such time series prediction problems, older training points are associated with lower weights, so that the effect of older training points is reduced when the regression function is optimized.

In sequential learning and inference methods such as time series problems, where a point from the recent past may be given greater weight than a point from further in the past, function of timeti can be selected as the weighted SVM scheme. Lin and Wang [31] proposed three time functions, linear, quadratic, and exponential, as shown in Eqs. (6)-(8). Those three time functions were used by Khemchandaniet al. [32] on financial time series forecasting problems, who demonstrated their abilities to bring about better results than SVM.

$$
\begin{aligned}
& \mathrm{Si}=\mathrm{f}_{\mathrm{l}}\left(\mathrm{t}_{\mathrm{i}}\right)=\frac{1-\sigma}{\mathrm{t}_{\mathrm{m}}-\mathrm{t}_{1}} \mathrm{t}_{\mathrm{i}}+\frac{\mathrm{t}_{\mathrm{m}} \sigma-\mathrm{t}_{1}}{\mathrm{t}_{\mathrm{m}}-\mathrm{t}_{1}} \mathrm{t}_{\mathrm{i}} \\
& S i=f_{q}\left(\mathrm{t}_{\mathrm{i}}\right)=(1-\sigma)\left(\frac{t_{i}-t_{1}}{t_{m}-t_{1}}\right)^{2}+\sigma \\
& S i=f_{e}\left(\mathrm{t}_{\mathrm{i}}\right)=\frac{1}{1+\exp \left(\sigma-2 \sigma\left(\frac{t_{i}-t_{1}}{t_{m}-t_{1}}\right)\right)}
\end{aligned}
$$

However, as the wSVM was developed from SVM, it presents the user with similar problems. Schlkopf and Smola (2002) expressed that SVM bandwidth and penalty parameter $\mathrm{C}$, which determines the trade-off between margin maximization and violation error minimization, represent an issue that requires attention and handling. Another point of concern is the setting of kernel parameters, such as gamma $(\gamma)$, on the radial basis function, which must also be set properly to improve prediction accuracy. In addition, using wSVM requires users to set a further parameter, i.e., weighting data parametero. Therefore, three different parameters must be optimized, including the penalty parameter $(\mathrm{C})$, kernel parameter (i.e. $\gamma$, if the RBF kernel is employed), and $\sigma$. To overcome this challenge, an optimization technique (e.g.,
fmGA) may be used to identify best parameters simultaneously [24]

\section{EXPERIMENTAL RESULTS AND DISCUSSION}

\section{Database}

This section verifies and validates the performance of our system using WSVM in predicting HPC compressive strength. The model proposed herein predicts the compressive strength of HPC using an experimental database originally collected by Yeh [13] and furnished from various university research labs, which was posted to the University of California, Irvine machine learning repository website. The database includes a total of 1030 concrete samples and covers 9 attributes, 8 of which are quantitative input variables and 1 of which is an output variable. Each instance includes the amount of cements, fly ash, blast furnace slag, water, superplasticizers, coarse aggregate, fine aggregate, age of testing and the compressive strength (in $\mathrm{MPa})$.

Table 2 shows the general details of the nine attributes used in this study. However, the database often contains unexpected inaccuracies [24], as for instance, the class of fly ash may not be indicated.

Another problem is related to superplasticizer as chemical admixture produced by different manufactures which may have different chemical compositions [6,20]. Moreover, Chou et al. [6] identified that such inaccuracies induce another difficulty related to the compressive strength which can be classified into a specific class such as high or low concrete compres sive strength.

The HPC database often contains inaccuracies due to mixing proportions, mixing techniques and ingredient characteristics (e.g., varying degrees offinesses, classes offly ash, and types of superplasticizer). Such makes prediction of HPC compressive strength a highly uncertain task. Also HPC databases contains compressive strength measures representing 14 different testing ages ranging from 1 day to 365 days as shown in Table 3 )

\section{Experiment Setup}

The experiments were carried out in Matlab (R2012a), on a 64-bit, PC with an i5 microprocessor with 4 cores, 4 GB of RAM and a hard disk of 250 GB.

To develop the HPC compressive strength prediction system, the 1030 samples were divided randomly into training and testing sets $90 \%$ or 927 samples were assigned to the training set and the remainder, $10 \%$ or 103 samples, were assigned to the testing set. As the wSVM was to be compared against SVM result accuracies, SVM parameter setting procedures followed previous researcher 
suggestions and settings. In this study, as suggested by $\mathrm{Hsu}$ et al. [33] parameter settings for SVMs, herein $\mathrm{C}$ and $\gamma$ were set to 1 and $1 / \mathrm{k}$ respectively, with $\mathrm{k}$ representing number of input patterns. This study employed four performance measures, namely root mean square error (RMSE), coefficient correlation (r), coefficient of determination (R2) and mean absolute error (MAE) to verify and validate the accuracy of the proposed system and other AI models. Table 4 shows RSME, r, R2, and MAE results of the proposed wSVM system (linear, quadratic and exponential time series functions) compared against SVM.

\section{Results and discussion}

This section presents the results of comparing wSVM to other prediction technique including such as SVM.

The performance of the proposed system was evaluated by three statistical measures namely root mean square error (RMSE), coefficient of determination (R2) and mean absolute error (MAE) to verify and validate the accuracy of the proposed method

$$
\begin{aligned}
& \mathrm{MAE}=\frac{1}{n} \sum_{i=1}^{n}\left|P_{i}-A_{i}\right| \\
& \mathrm{RMSE}=\sqrt{\frac{1}{n} \sum_{i=1}^{n}\left(P_{i}-A_{i}\right)^{2}} \\
& R^{2}=1-\frac{\sum_{i=1}^{n}\left(A_{i}-P_{i}\right)^{2}}{\sum_{i=1}^{n}\left(P_{i}-M\right)^{2}}
\end{aligned}
$$

Here, $\mathrm{Ai}=$ Actual value, $\mathrm{M}=$ mean of actual value; $\mathrm{Pi}=$ Predicted value; $\mathrm{n}=$ nu mber of data $(1,2$, $3 \ldots)$.

Table 4 shows RSME, R2, and MAE results of the proposed system using wSVM with polynomial and RBF kernel functions compared against the other AI systems (SVM and BPN)

Based on the three different evaluation methods for both training and testing datasets, wSVM provided the best satisfactory result. In comparing SVM based on RSME and MAE, SVM performed slightly better than wSVM, but only on training data (not on the testing data set). However, in terms of coefficient of determination (R2) for the training data set, wSVM is comparable to SVM. Fig. 2 presents scatter diagrams of SVMs and wSVM using polynomial and RBF (Radial basis Function) kernels for the training data set. Better results were achieved by wSVM in terms of predicting testing dataset results, which shows that the wSVM training data learning process provides a prediction model superior to SVM. Such confirms that wSVM (polynomial ann RBF kernels) delivers comparable or higher performance than SVM.
This better learning ability demonstrates wSVM ability to cope with uncertain characteristics inherent in HPC databases. Moreover wSVM model is also able to map the complex relationship between input and output variables as well as manage time series characteristics inherent to HPC databases. While wSVM employed three different time series functions (linear, quadratic and exponential) to weigh data points, one preferable time series function should be chosen based on performance achieved by each time series function, both in the training and testing datasets. As shown in Table 4, the wSVM using quadratic functions generally provides slightly better performance, especially on the testing data set, in comparison with the wSVM using polynomial and RBF kernel functions. However, it should be noted that differences in performance obtained between the two kernel functions were not significant. This shows that there remains room for improvement to find a better time series function to predict HPC compres sive strength. The proposed model, wSVM, offers the potential to predict HPC compressive strength. The practitioners can obtain early, applicable and reliable prediction of concrete compressive strength for pre-design and quality control, as waiting 28 days to get 28 -day compressive strength or later-age compressive strength is time-consuming. In accordance with Zain and Abd [34] and Chou et al. [35] the rapid prediction would enable the adjustment of mix proportion to avoid situation where concrete does

\begin{tabular}{|c|c|c|c|}
\hline Input/output variables & Unit & Vinimum & Maximum \\
\hline Cement & $(\mathrm{kg} / \mathrm{m} 3)$ & 102.00 & 540.00 \\
\hline Blast funnace slag & $(\mathrm{kg} / \mathrm{m} 3)$ & 0.00 & 359.40 \\
\hline Fly ash & $(\mathrm{kg} / \mathrm{m} 3)$ & 0.00 & 200.10 \\
\hline Water & $(\mathrm{kg} / \mathrm{m} 3)$ & 121.75 & 247.00 \\
\hline Superplasticizer) & $(\mathrm{kg} / \mathrm{m} 3)$ & 0.00 & 32.20 \\
\hline Coarse aggregate) & $(\mathrm{kg} / \mathrm{m} 3)$ & 801.00 & 1145.00 \\
\hline Fine ageregate & $(\mathrm{kg} / \mathrm{m3})$ & 594.00 & 992.60 \\
\hline Age of testing & Day & 1.00 & 365.00 \\
\hline Concrete compressive strength & $\mathrm{MPa}$ & 2.33 & 82.60 \\
\hline
\end{tabular}
not reach the required compressive strength, which would save time and construction costs.

Table I

HPC database: input and output variables

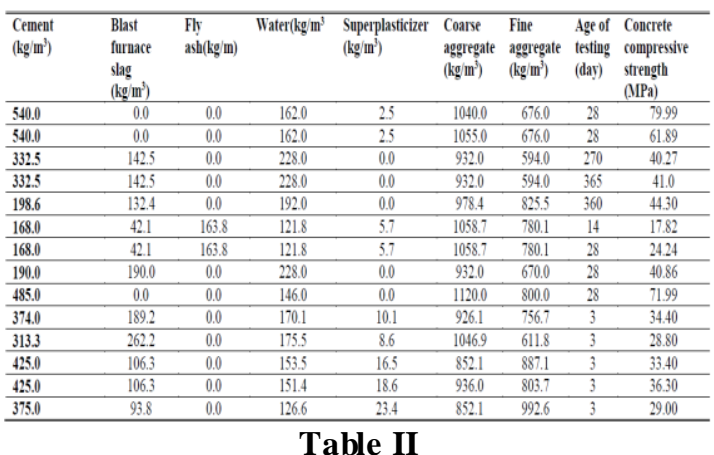

HPC database Examp les 


\begin{tabular}{|c|c|c|c|c|c|}
\hline \multirow[t]{2}{*}{ Dataset } & \multirow{2}{*}{$\begin{array}{l}\text { Evaluation } \\
\text { performance } \\
\text { measurement }\end{array}$} & \multicolumn{2}{|l|}{ SVMs } & \multicolumn{2}{|l|}{ wSVMs } \\
\hline & & polynomial & RBF & \begin{tabular}{|l} 
Polynomial \\
\end{tabular} & $\mathrm{RBF}$ \\
\hline \multirow[t]{3}{*}{ Training set } & $\mathrm{R}^{2}$ & 71.23 & 82.35 & 90.62 & 92.23 \\
\hline & MAE (Npa) & 7.59 & 6.87 & 5.23 & 5.34 \\
\hline & RMSE (Mpa) & 8.62 & 5.65 & 5.49 & 5.68 \\
\hline \multirow[t]{3}{*}{ Testing set } & $\mathrm{R}^{2}$ & 73.98 & 84.34 & 91.95 & 93.36 \\
\hline & MAE (Mpa) & 8.76 & 6.49 & 5.89 & 5.89 \\
\hline & RMSE (Mpa) & 9.76 & 6.87 & 6.34 & 6.03 \\
\hline
\end{tabular}

\section{Table III}

Comparison of results using SVMs and weighted SVMs (Polynomial and RBF kernels)
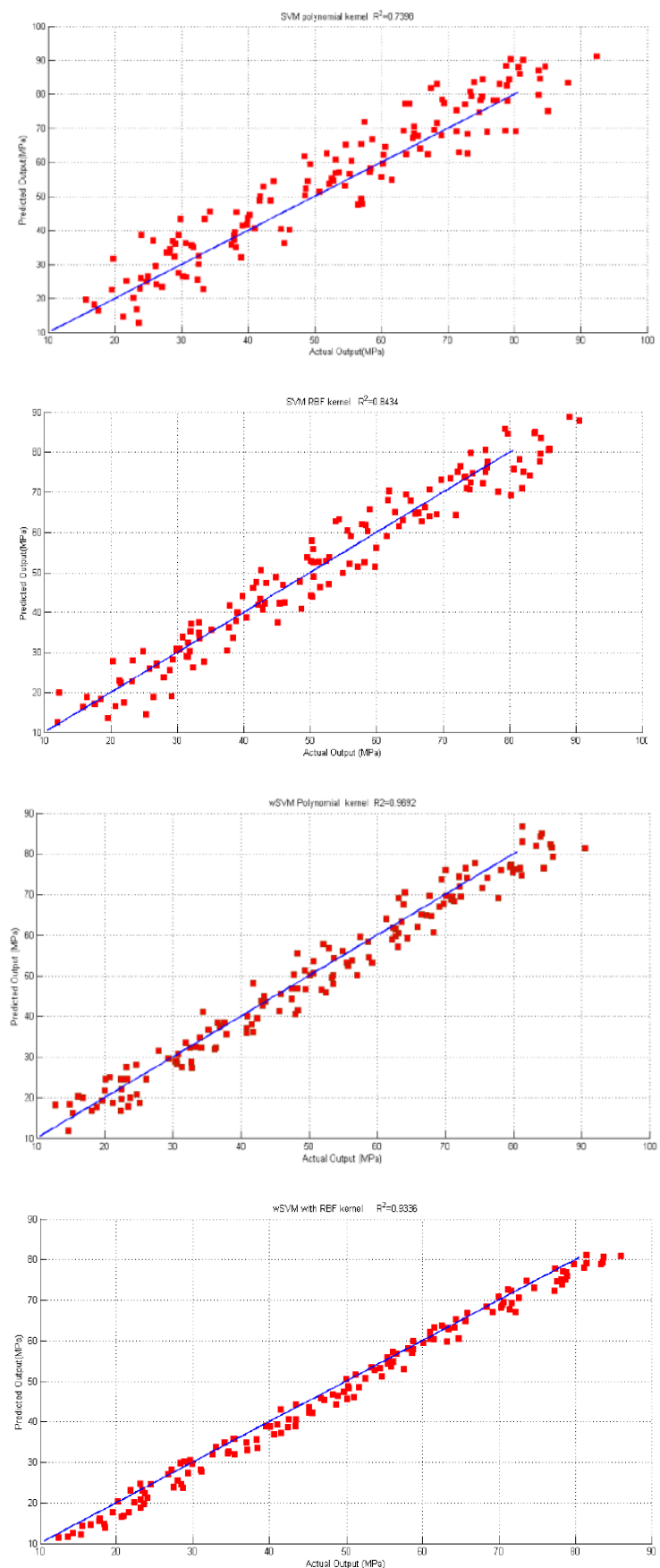

Fig. 2

Scatter diagrams of SVMs and wSVM (Polynomial and RBF kernels)

\section{CONCLUSION}

This paper proposed wSVM as a hybrid AI system to predict HPC compressive strength, a mechanical property critical to measuring HPC quality. wSVM was developed by fusing FL. Therefore wSVMs was used to address uncertainties inherent in HPC and to deal with complex relationships related to fuzzy input-output mapping data in the HPC database (e.g., compressive strength) with regard to testing age. In comparison with SVMs, the accuracy of the proposed WSVM was significantly better for different evaluation measurements.

Such results demonstrate the superior ability of wSVM to manage 1) time series data characteristics inherent in HPC experimental data, 2) complex relationships between input and output variables, and 3) uncertainties inherent in HPC databases. Therefore, wSVM offers strong potential as a predictive tool for HPC compressive strength.

\section{REFERENCES}

[1]. I.C. Yeh, Modelling of strength of highperformance concrete using artificial neural networks, Cement and Concrete Research 28 (12) (1998) 1797-1808.

[2]. Mousavi, S. M., A minian, P., Gandomi, A. H., Alavi, A. H., \& Bolandi, H. (2012). A new predictive model for compressive strength of HPC using gene expression programming. Advances in Engineering Software, 45(1), 105-114.

[3]. Peng, C. H., Yeh, I. C., \& Lien, L. C. (2010). Building strength models for highperformance concrete at different ages using genetic operation trees, nonlinear regression, and neural networks. Engineering with Computers, 26(1), 61-73.

[4]. Bharatkumar, B. H., Narayanan, R., Raghuprasad, B. K., \& Ramachandramurthy, D. S. (2001). Mix proportioning of high performance concrete. Cement and concrete composites, 23(1), 71-80

[5]. C.H. Lim, Y.S. Yoon, J.H. Kim, Genetic algorithm in mix proportioning ofhighperformance concrete, Cement and Concrete Research 34 (2004) 409-420.

[6]. Chou, J. S., \& Tsai, C. F. (2012). Concrete compressive strength analysis using a combined classification and regression technique. Automation in Construction, 24, $52-60$.

[7]. ACI Committee 363. (1984, July). State of the Art Report on High-Strength Concrete. In ACI Journal Proceedings (Vol. 81, No. 4). ACI.

[8]. P.C. Aitcin, High Performance Concrete, E\&FN SPON, New York, 1998. 
[9]. Mehta, P. K., \& Aitcin, P. C. (1990). Microstructural basis of selection of materials and mix proportions for high-strength concrete. ACI Special Publication, 121.

[10]. A.I. Laskar, S. Talukdar, A new mix design method for high performance concrete, Asian Journal of Civil Engineering (Building and Housing) 9 (1) (2008) 15-23.

[11]. Arhras, G., \& Foo, H. C. (1994). A knowledge-based system for selecting proportions for normal concrete. Expert systems with applications, 7(2), 323-335.

[12]. C.H. Lim, Y.S. Yoon, J.H. Kim, Genetic algorithm in mix proportioning ofhighperformance concrete, Cement and Concrete Research 34 (2004) 409-420.

[13]. J. Kasperkiewicz, J. Racz, A. Dubrawski, HPC strength prediction using artificial neural network, Journal of Computing in Civil Engineering 9 (4) (1995)279-284.

[14]. M.F.M. Zain, S.M. Abd, Multiple regression model for compressive strength prediction of high performance concrete, Journal of Applied Science 9 (1) (2009) 155-160.

[15]. Alilou, V. K., \& Teshnehlab, M. (2010). Prediction of 28-day compressive strength of concrete on the third day using artificial neural networks. International Journal of Engineering, 3(6), 565-576.

[16]. ACI Committee 211, 1991 "Standard Practice for selecting properties for normal, heavy weight concrete", (ACI211.1-91) American Concrete Institute, Detroit.Chengju G., 1989. "Maturity of concrete: Method for predicting early stage strength". ACI Materials Journal, Vol.86 (4), pp. 341-353.

[17]. Oluokun F.A., Burdette E.G., Harold Deatherage J., 1990. "Early-age concrete strength prediction by maturity - another look". ACI Materials Journal, Vol. 87(6), pp. 565-572.

[18]. Popovics S., 1998. "History of a mathematical model for strength development of Portland cement concrete". ACI Materials Journal, Vol. 95(5), pp. 593-600.

[19]. H.G. Ni, J.Z. Wang, Prediction of compressive strength of concrete by neural networks, Cement and Concrete Research 30 (2000) 1245-1250.

[20]. I.C. Yeh, L.C. Lien, Knowledge discovery of concrete material using genetic operation trees, Expert System with Applications 36 (2009) 5807-5812.

[21]. S. Akkurt, S. Ozdemir, G. Tayfur, B. Akyol, The use of GA-ANNs in the modeling of compressive strength of cement mortar, Cement and Concrete Research 33(2003) 973-979.
[22]. Mousavi, S. M., A minian, P., Gandomi, A. H., Alavi, A. H., \& Bolandi, H. (2012). A new predictive model for compressive strength of HPC using gene expression programming. Advances in Engineering Software, 45(1), 105-114.

[23]. BURGES, Christopher JC. A tutorial on support vector machines for pattern recognition. Data mining and knowledge discovery, 1998, vol. 2, no 2, p. 121-167

[24]. Cheng, M. Y., \& Wu, Y. W. (2009). Evolutionary support vector machine inference system for construction management. Automation in Construction, 18(5), 597-604.

[25]. GUPTA, S. M. Support vector machines based modelling of concrete strength. World Academy of Science, Engineering and Technology, 2007, vol. 36, p. 305-311.

[26]. Lin, C. F., \& Wang, S. D. (2002). Fuzzy support vector machines. Neural Networks, IEEE Transactions on, 13(2), 464-471.

[27]. I.C. Yeh, Modelling of strength of highperformance concrete using artificial neural networks, Cement and Concrete Research 28 (12) (1998) 1797- 1808.

[28]. Hutchinson, T. C., \& Chen, Z. (2006). Improved image analysis for evaluating concrete damage. Journal of Computing in Civil Engineering.

[29]. Fan, H., \& Ramamohanarao, K. (2005, July). A weighting scheme based on emerging patterns for weighted support vector machines. In Granular Computing, 2005 IEEE International Conference on (Vol. 2, pp. 435-440). IEEE.

[30]. Vapnik, V. (2013). The nature of statistical learning theory. Springer Science \& Business Media.

[31]. C.F. Lin, S.D. Wang, Fuzzy support vector machines, IEEE Transactions on Neural Networks 13 (2) (2002) 464-471.

[32]. Khemchandani, R., \& Chandra, S. (2009). Regularized least squares fuzzy support vector regression for financial time series forecasting. Expert Systems with Applications, 36(1), 132-138.

[33]. Hsu, C. W., Chang, C. C., \& Lin, C. J. (2003). A practical guide to support vector classification.

[34]. Zain, M. F. M., \& Abd, S. M. (2009). Multiple regression model for compressive strength prediction of high performance concrete. Journal of applied sciences, 9(1), 155-160.

[35]. Cheng, M. Y., Chou, J. S., Roy, A. F., \& Wu, Y. W. (2012). High-performance concrete compressive strength prediction using time- 
weighted evolutionary fuzzy support vector machines inference model. Automation in Construction, 28, 106-115.

\begin{abstract}
Authors' infor mation
${ }^{1}$ LETI Laboratory, EHTP Casablanca

Rguig Mustapha received the Master's degree (DEA) from Louis Pasteur University and PHD degree in Civil Ingineering from Nantes University in 2001 and 2005, respectively. He is currently a professor at the Department of civil engineering, EHTP Casablanca.

Mohamed El AROUSSI received his Bachelors degree (License es Sciences) in science computing automatic electronic electrotechnic, his Masters degree (DESA) in computer science and telecommunication from the Faculty of Science, University Mohamed V-Agdal, Rabat, Morocco, in 2004 and his Ph.D. degree in the University of Mohammed V-Agdal, Rabat, Morocco, in 2009. He is currently a professor at the Department of electrical engineering, EHTP Casablanca.
\end{abstract}

